\title{
The future of meniscus science: international expert consensus
}

\author{
Nicholas N. DePhillipo ${ }^{* *}$, Robert F. LaPrade², Stefano Zaffagnini ${ }^{3}$, Caroline Mouton ${ }^{4,5}$, Romain Seil ${ }^{4,5,6}$ and \\ Philippe Beaufils ${ }^{7}$
}

\begin{abstract}
Purpose: The purpose of this study was to evaluate the main focus areas for research and development for furthering the state of meniscus science in 2021.

Methods: An electronic survey including 10 questions was sent in a blind fashion to the faculty members of the $5^{\text {th }}$ International Conference on Meniscus Science and Surgery. These faculty served as an expert consensus on the future of research and development areas of meniscus science. Survey responses were analyzed using descriptive statistics and ranking weighted averages were calculated to score survey questions.

Results: Of the 82 faculty, 76 (93\%) from 18 different countries completed the survey (84\% male, 16\% female). The highest ranked future research and development focus areas were meniscus repair, biologics, osteotomy procedures, addressing meniscus extrusion, and the development of new therapies for the prevention of posttraumatic osteoarthritis. Currently, the most'valuable' type of biologic reported for meniscus treatment was platelet-rich plasma. The main reported global research limitation was a lack of long-term clinical outcomes data. The most promising emerging medical technologies for improving meniscus science were 3-D printing, personalized medicine, and artificial implants.
\end{abstract}

Conclusions: This survey suggests that the future of meniscus science should be focused on meniscal preservation techniques through meniscus repair, addressing meniscal extrusion, and the use of orthobiologics. The lack of longterm clinical outcomes was the main reported research limitation globally for meniscus treatment. Future product development utilizing emerging medical technologies suggest the use of 3-D printing for meniscal transplants/scaffolds, personalized treatment, and bioengineering for artificial implants.

Level of Evidence: Level V.

Keywords: Meniscus repair, Orthobiologics, Meniscal extrusion, Knee osteotomy, Biomechanics, Posttraumatic osteoarthritis, Orthopedic medical devices

\footnotetext{
*Correspondence: Nickd.atc@gmail.com

'Oslo Sports Trauma Research Center, 4014 Ulleval Stadion, 0806 Oslo,

Norway

Full list of author information is available at the end of the article
}

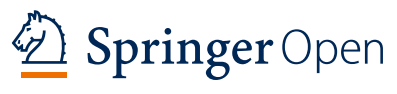

(c) The Author(s) 2021. Open Access This article is licensed under a Creative Commons Attribution 4.0 International License, which permits use, sharing, adaptation, distribution and reproduction in any medium or format, as long as you give appropriate credit to the original author(s) and the source, provide a link to the Creative Commons licence, and indicate if changes were made. The images or other third party material in this article are included in the article's Creative Commons licence, unless indicated otherwise in a credit line to the material. If material is not included in the article's Creative Commons licence and your intended use is not permitted by statutory regulation or exceeds the permitted use, you will need to obtain permission directly from the copyright holder. To view a copy of this licence, visit http://creativecommons.org/licenses/by/4.0/. 


\section{Background}

The advancement of meniscus treatment through scientific research can be attributed to the current success of clinical treatment strategies for patients with meniscusassociated pathologies. Understanding the structure and function of the meniscus has played a pivotal role in the evolution of meniscus science $[1,16]$. In 1884, Sutton referred to the menisci as 'functionless' remnants of intra-articular leg muscles [48]. In 1948, the first degenerative changes of the knee joint following meniscectomy were reported by Fairbank [11]. By 1982, Arnoczky and Warren recognized that the menisci were one of the 'most important' structures determining the future of the knee joint [1]. This century-long transition of the conceptualization of the meniscus from being 'functionless' to 'most important' was through the evolution of scientific research and innovation, particularly involving the discovery of the increased risk of joint degeneration associated with the loss of meniscus tissue $[6,39]$.

The state of meniscus science has advanced immensely with the evolution of scientific technologies, such as the advent of the arthroscope, which has led to an improved understanding of the meniscus for the longevity of the knee joint $[17,50]$. As a result of both technological and research advancements, a paradigm shift from meniscus resection to meniscus repairs was established $[2,36,45]$. The goal of any technological evolution is to meet the needs and expectations of its users - this is the same goal with orthopaedic surgery and the evolution of meniscal treatment [3]. Thus, understanding the key focus areas for future research and development may lead to accelerated technological innovation in the field of meniscus science $[10,26]$. Therefore, the purpose of this study was to evaluate the main focus areas for research and development for furthering the state of meniscus science in 2021. We accomplished this by surveying a sample of the top scientific experts from an international consensus on meniscus science.

\section{Methods}

\section{Survey development}

This study was approved by the organizing committee members of the $5^{\text {th }}$ International Conference on Meniscus Science and Surgery prior to being conducted. An electronic survey including 10 questions was sent in a blind fashion to all of the faculty members of the $5^{\text {th }}$ International Conference on Meniscus Science and Surgery (Supplement 1). These faculty were primarily orthopedic surgeons. The survey questions were developed by the current authors according to previous trends in the literature regarding meniscus science and also by expert opinionated knowledge from years of clinical practice. A cover letter that accompanied the survey stated the purpose of the survey and ensured anonymity. All contacted participants had the opportunity to decline the survey. The survey was sent out and responses were collected from October 2020 to December 2020.

Therapies for the prevention of posttraumatic osteoarthritis (PTOA) were defined as any intervention designed to avert or avoid OA development in high-risk patient populations. Symptom resolution therapies were defined as any therapy focused on reducing the symptomatology of patients with existing OA, including pain, stiffness, swelling, joint range-of-motion, muscle weakness, fatigue, joint instability, and pain-related psychological distress. Disease-modifying therapies were defined as any treatment that focused on retardation of OA (slowing the speed of progression), a complete halt in disease progression, or a reversal in disease progression (regeneration of targeted tissue).

\section{Statistical analysis}

Data were prospectively collected via an online survey tool (www.surveymonkey.com). They were extracted from the online survey database and summarized. Standard descriptive statistics were performed. Certain focus areas were ranked (low to high) among the respondents from a score of 1 ('Not Helpful') to a score of 5 ('Most Helpful'). Weighted averages were then calculated to provide a statistical datapoint for these questions. For questions that were not weighted, multiple answers were allowed and thus the sum of such questions was not equal to $100 \%$.

\section{Results}

Of the 82 faculty members, 76 (93\%) from 18 different countries completed the survey ( $84 \%$ male, $16 \%$ female). Sixty-four (84\%) individuals of the expert panel were orthopaedic surgeons while $12(16 \%)$ were scientists/ physiotherapists. The top 3 ranked focus areas of research and development for furthering the state of meniscus science were: meniscus repair (weighted average: 4.55), biologics (weighted average: 4.15), and surgical medical devices (weighted average: 4.00) (Table 1). Studying clinical outcome parameters (64\%) was reported as the most 'important' focus area for improving clinical outcomes for meniscus repair. The reported most 'valuable' biologic for meniscus treatment was autologous blood (25\%), including platelet-rich plasma (PRP). The reported least 'valuable' biologic for meniscus treatment was amniotic fluid $(0 \%)$. However, the majority of respondents $(28 \%)$ reported that none of the current biologics were 'valuable' for meniscal treatment (Fig. 1).

Addressing meniscal extrusion (52\%) was reported as the main focus area for improving meniscal allograft transplantation (MAT), followed by improved patient 
Table 1 Primary future research and development focus areas with weighted averages ${ }^{a}$

\begin{tabular}{ll}
\hline Biologics & 4.15 \\
Surgical medical devices & 4.00 \\
Meniscus engineering & 3.84 \\
Meniscus transplantation & 3.63 \\
Nonsurgical medical devices & 2.63 \\
Meniscus repair & 4.55
\end{tabular}

a Ranking weighted averages: (1) not helpful—(2) low yield—(3) average yield - (4) moderate yield—(5) most helpful

selection (42\%) and the utilization of biologics for improved graft incorporation and healing (42\%). Osteotomy procedures (weighted average: 4.13), MAT (weighted average: 4.10 ), and biologics (weighted average: 3.83 ) were the top 3 ranked focus areas for treating patients with meniscal deficiency. Regarding meniscal preservation, meniscus repair (weighted average: 4.66), intra-articular repair devices (weighted average: 4.16), and biologics (weighted average: 3.97 ) were reported as the top ranked focus areas. The top ranked focus areas for treating patients with meniscus-associated PTOA were surgery (osteotomy, MAT, cartilage resurfacing; 64\%), biologics (55\%), pharmaceuticals (27\%) and nonsurgical medical devices (unloader bracing and supports; 27\%).

The main focus area for developing new therapies in treating patients with meniscus-associated PTOA was prevention (64\%), followed by the development of disease-modifying drugs $(24 \%)$ and symptomatic management therapies (12\%). The main current global research limitations for improving clinical outcomes in meniscus tear/deficient patient populations were lack of long-term clinical outcomes data (55\%), lack of funding (33\%), and lack of understanding the clinical problem (33\%) (Fig. 2). The use of 3-D printing for meniscal transplants/scaffolds (61\%), personalized medicine (52\%), and artificial implants (including bioengineering, nanoparticles, and synthetic devices; $43 \%$ ) were reported as the top ranked emerging medical technologies to have the greatest impact for furthering meniscus science (Fig. 3).

\section{Discussion}

The most important findings of this survey were that the highest ranked future research and development focus areas included meniscus repair, biologics, osteotomy procedures, addressing meniscus extrusion, and the development of new therapies for the prevention of PTOA. Currently, the reported most 'valuable' type of biologic for meniscus treatment was PRP, while amniotic fluid was reported as least 'valuable.' The main reported global research limitation was lack of long-term clinical outcomes data. Finally, the most promising emerging medical technologies for improving meniscus science in the twenty-first century were 3-D printing, personalized medicine, and bioengineering of artificial implants.

This meniscus expert panel collectively suggests focusing future research and development on meniscal preservation through meniscus repair and the use of orthobiologics. Recent evidence including basic science and expert consensus statements have led to an improved understanding of treating meniscal lesions and use of biologic augmentation [4, 14, 20, 26, 28, 36, 40, 45, 47]. Both clinical outcomes data and animal models indicate promising results for meniscal repair and the potential for improved meniscal healing with biologic augmentation [23, 31, 35, 37]. Additionally, this survey suggests that future research and development should focus on intra-articular devices to further improve meniscal repair and enhance the delivery and sustainability of biologics inside the knee joint. Previous research has demonstrated the evolution of meniscal repair strategies, evident by the transition from inside-out techniques to all-inside repair devices [43, 49]. Continual development of biomedical devices involving meniscus repair and biologics delivery are of high importance based on this expert panel survey.

This study demonstrated that biologics, specifically PRP, were consistently ranked as a leading focus area

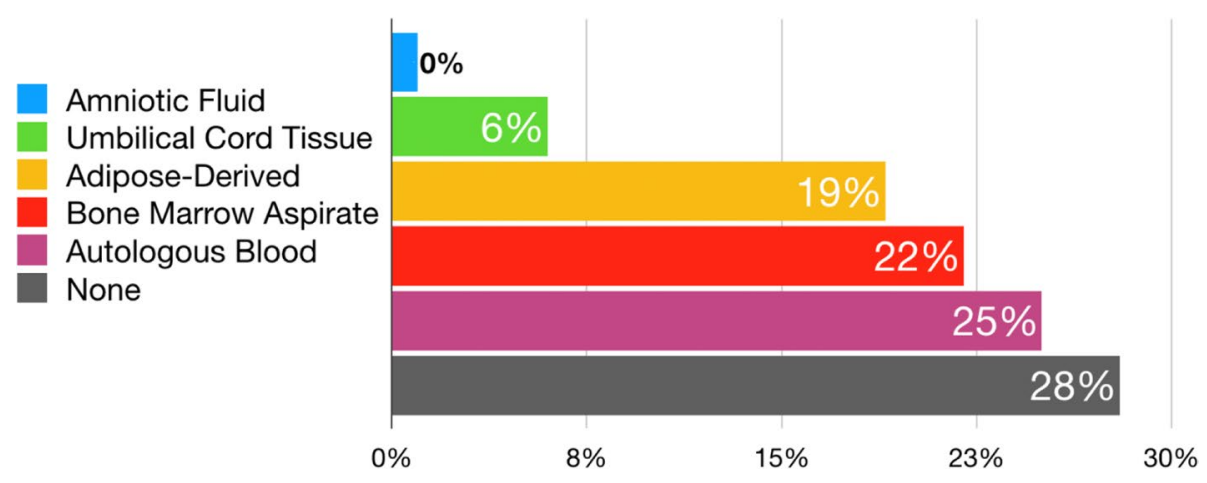

Fig. 1 Type of biologics reported as currently 'most valuable' for meniscus treatment 


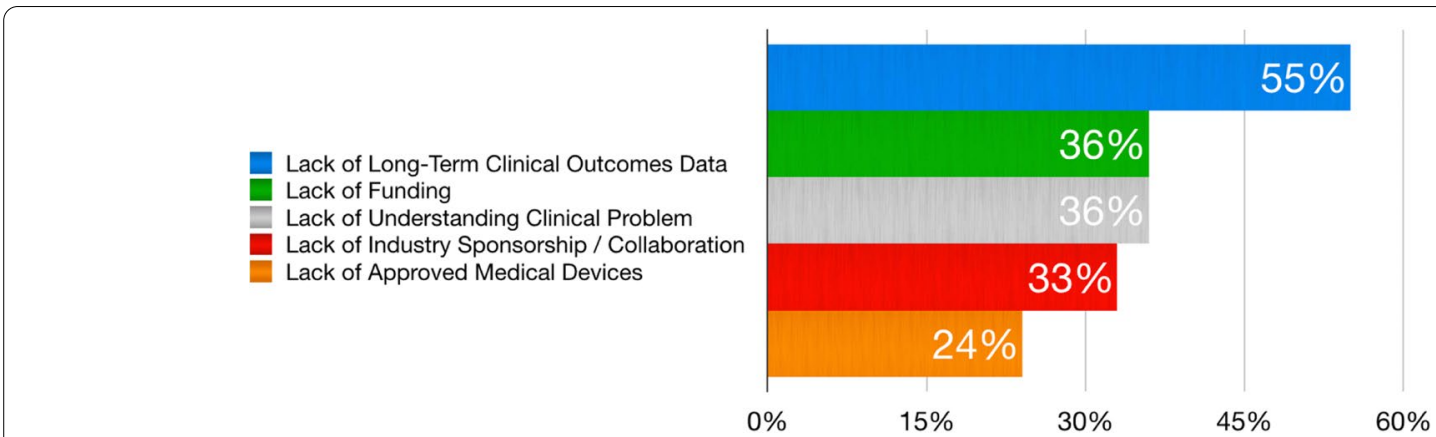

Fig. 2 Current global research limitations for improving clinical outcomes in meniscal tear/deficient patient populations

within each domain of meniscus science. This coincides well with current clinical practice apparent by the expanding use and global interest in utilizing biologics for treating an array of musculoskeletal disorders [34, 35, 42, 44, 47]. However, despite the increased interest and consensus for more research and development of biologics in meniscus science, the majority of respondents in this survey reported that biologics are currently not valuable for the treatment of meniscal injuries. This criticism is perhaps due to the unproven regenerative capacity of orthobiologics in clinical studies, despite promising evidence in basic science and animal models [31, 35, 44, 47, 52]. Therefore, the question remains "how" to advance from a technology development perspective. This should involve parallel discovery among clinicians and scientists with collaboration and investment from both the public and private sector.

While new discoveries and sophisticated research methods will undoubtedly continue to contribute to the evolution of orthobiologics, the clinical utility of such therapeutics remain partially limited due to the limited cellular manipulation of biologic products for human use [22, 32, 33]. Therefore, one technological approach for improving the clinical efficacy of biologics in meniscus science is through the development of novel drug delivery platforms. Currently, the major challenges affecting successful delivery of biologics within joints includes rapid clearance of drugs due to passive release and lack of response to the body's natural physiologic loading mechanisms [38]. Consequently, self-regulating drug delivery systems designed specifically for the mechanical environment of musculoskeletal tissues wherein physiologic feedback actively controls release kinetics have been developed as a solution to overcome this clinical barrier [29]. Controlled drug delivery for musculoskeletal environments show promise in a variety of orthopaedic conditions, including meniscal tears and the consequential degenerative cascade of PTOA [7, 25, 30].

This survey demonstrated high interest in the development of preventative therapies in patients with meniscusassociated PTOA. It is known that there is currently no treatment strategy that can prevent the progression of OA after injury and many treatment options may provide only partial symptomatic relief [41]. The clinical need for improved treatments in patients with meniscus tears and PTOA is clearly evident $[12,18,20,23,24]$; meanwhile the technology seeds in bioengineering show great promise for meniscus application in early development phases [13, 15, 21, 27, 29, 51]. Therefore, combining both a clinical need- and technology seed-driven approach may allow for accelerated innovation $[8,9,19]$, especially in the domain

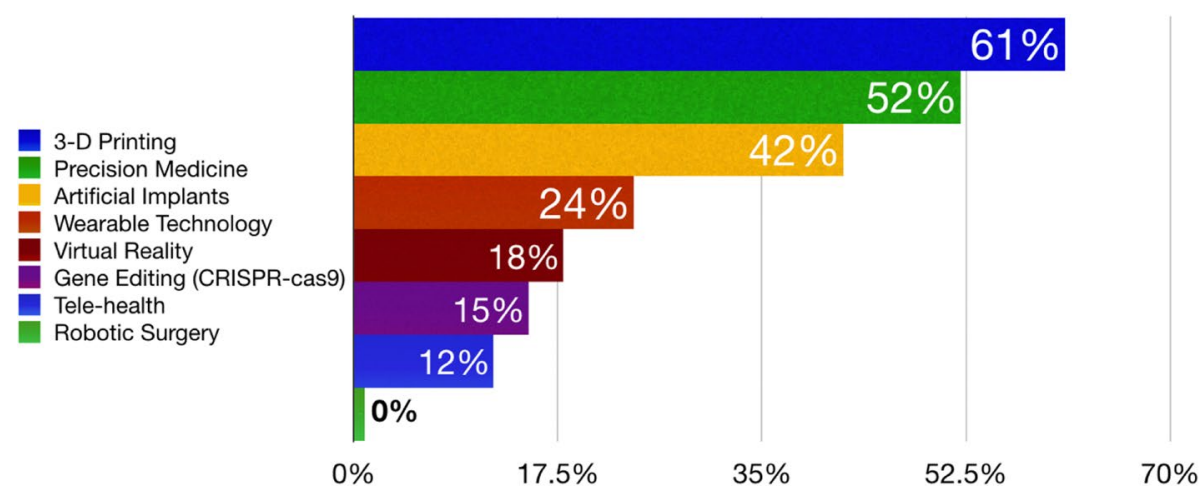

Fig. 3 Top ranked emerging medical technologies to have the greatest impact for furthering meniscus science 
of meniscus science and knee PTOA. Consequently, future research aimed to optimize biologically-targeted delivery systems may improve the efficacy of current orthobiologics while also assisting in the prevention of knee PTOA. Yet, preserving the meniscus through surgical repair (when indicated) remains the number one priority $[12,18,23,40,45]$.

Addressing meniscal extrusion was a key focus area for research and development as indicated by this meniscus expert panel. Prior research has shown meniscal extrusion to be a determinant of success for corrective surgery in both meniscal repair and transplantation. Specifically, by addressing meniscal extrusion and successfully relocating or recentering the meniscus in its native anatomic position may best restore the load bearing and force reduction functions of the meniscus. Future research may focus on improving surgical techniques and developing medical technologies that allow for enhanced fixation of the meniscus to address this joint extrusion.

Currently, the main global research limitation in the field of meniscus science is the lack of long-term clinical outcomes as reported by the expert panel. This survey suggests the need for long-term monitoring programs of meniscal tear patients including registry data and multi-center, international collaborations. The future of clinical care is not only dependent on emerging technology, such as 3-D printing and artificial implants, but also relies heavily on interdisciplinary collaboration and innovation $[4,5,14,27,46]$.

There were limitations of this study inherent to that of a survey. Therefore, the subjective perspectives of the survey respondents cannot be validated with evidence-based recommendations. Furthermore, these practices may be adopted into future research studies for validation. Additionally, there may be inherent bias introduced as a result of an opinionated survey from primarily orthopedic surgeons, thus the results should be interpreted with caution.

\section{Conclusions}

This survey suggests that the future of meniscus science should be focused on meniscal preservation techniques through meniscus repair, addressing meniscal extrusion, and the use of orthobiologics. The lack of longterm clinical outcomes is the main reported research limitation globally for meniscus treatment. Future product development utilizing emerging medical technologies suggest the use of 3-D printing for meniscal transplants/scaffolds, personalized treatment, and bioengineering for artificial implants.

\section{Abbreviations}

PTOA: Posttraumatic osteoarthritis; PRP: Platelet-rich plasma; BMC: Bone marrow concentrate; MAT: Meniscus allograft transplant.

\section{Supplementary Information}

The online version contains supplementary material available at https://doi. org/10.1186/s40634-021-00345-y.

Additional file 1 Supplement 1 The survey questionnaire with responses from expert meniscus consensus*

\section{Acknowledgements}

The authors would like to thank and acknowledge the organizing committee members and faculty of the 2021 Meniscus Webinar.

\section{Authors' contributions}

All authors participated in the planning of the study and writing of the manuscript. NND conducted the survey and was involved with the study design, results interpretation, and writing of the manuscript. SZ provided oversight and contributed to writing of the manuscript. RFL contributed to conception and writing of the manuscript. CM was involved in data collection and participant recruitment. RS contributed to study design, results, and discussion. $\mathrm{PB}$ contributed to study design, results, and discussion. All authors read and approved the final manuscript.

Funding

The authors received no financial support for the research, authorship, and/or publication of this article.

Availability of data and materials

All data generated or analyzed in this study are included in this published article.

\section{Declarations}

\section{Ethics approval and consent to participate}

Consent was provided by survey respondents in writing via email and validated upon their approval.

\section{Consent for publication}

Not applicable.

\section{Competing interests}

The authors declare that they have no competing interests.

\section{Author details}

${ }^{1}$ Oslo Sports Trauma Research Center, 4014 Ulleval Stadion, 0806 Oslo, Norway. ${ }^{2}$ Twin Cities Orthopedics, Edina, MN, USA. ${ }^{3}$ Rizzoli Orthopedic Institutes of Bologna, Bologna, Italy. ${ }^{4}$ Department of Orthopaedic Surgery, Centre Hospitalier de Luxembourg, Clinique d'Eich, Luxembourg city, Luxembourg. ${ }^{5}$ Sports Medicine and Science, Luxembourg Institute of Research in Orthopaedics, Luxembourg city, Luxembourg. ${ }^{6}$ Human Motion, Orthopaedics, Sports Medicine and Digital Methods, Luxembourg Institute of Health, Luxembourg city, Luxembourg. ${ }^{7}$ Centre Hospitalier de Versailles, Versailles, France.

Received: 17 February 2021 Accepted: 15 March 2021

Published online: 31 March 2021

\section{References}

1. Arnoczky SP, Warren RF (1982) Microvasculature of the human meniscus. Am J Sports Med 10:90-95

2. Beaufils P, Pujol N (2017) Management of traumatic meniscal tear and degenerative meniscal lesions. Save the meniscus. Orthop Traumatol Surg Res 103:S237-S244

3. Beaufils P, Seil R, Becker R, Karlsson J, Menetrey J (2021) The orthopaedic community does not oppose the non-surgical treatment of degenerative meniscal lesions. Knee Surg Sports Traumatol Arthrosc 29:329-332 
4. Centeno CJ, Pastoriza SM (2020) Past, current and future interventional orthobiologics techniques and how they relate to regenerative rehabilitation: a clinical commentary. Int J Sports Phys Ther 15:301-325

5. Chae S, Lee SS, Choi YJ, Hong DH, Gao G, Wang JH et al (2021) 3D cellprinting of biocompatible and functional meniscus constructs using meniscus-derived bioink. Biomaterials 267:120466

6. Chambers HG, Chambers RC (2019) The natural history of meniscus tears. J Pediatr Orthop 39:S53-S55

7. Chen M, Guo W, Gao S, Hao C, Shen S, Zhang Z et al (2018) Biochemical stimulus-based strategies for meniscus tissue engineering and regeneration. Biomed Res Int 2018:8472309

8. Courvoisier A (2016) The future of academic innovation in the field of medical devices: is innovation still possible in orthopedics? Expert Rev Med Devices 13:807-813

9. Das S, Rousseau R, Adamson PC, Lo AW (2018) New business models to accelerate innovation in pediatric oncology therapeutics: a review. JAMA Oncol 4:1274-1280

10. DePhillipo NN, Engebretsen L, LaPrade RF (2019) Current trends among US surgeons in the identification, treatment, and time of repair for medial meniscal ramp lesions at the time of ACL surgery. Orthop J Sports Med 7:2325967119827267

11. Fairbank TJ (1948) Knee joint changes after meniscectomy. J Bone Joint Surg Br 30B:664-670

12. Faucett SC, Geisler BP, Chahla J, Krych AJ, Kurzweil PR, Garner AM et al (2019) Meniscus root repair vs meniscectomy or nonoperative management to prevent knee osteoarthritis after medial meniscus root tears: clinical and economic effectiveness. Am J Sports Med 47:762-769

13. Filardo G, Kon E, Perdisa F, Sessa A, Di Martino A, Busacca M et al (2017) Polyurethane-based cell-free scaffold for the treatment of painful partia meniscus loss. Knee Surg Sports Traumatol Arthrosc 25:459-467

14. Gilat R, Cole BJ (2020) Editorial commentary: orthobiologics-the evolution from symptom to structural modification in the treatment of articular cartilage defects. Arthroscopy 36:1441-1442

15. Guven E (2021) Nanotechnology-based drug delivery systems in orthopedics. Jt Dis Relat Surg 32:267-273

16. Hirschmann MT, Muller W (2015) Complex function of the knee joint: the current understanding of the knee. Knee Surg Sports Traumatol Arthrosc 23:2780-2788

17. Ikeuchi $H$ (1979) Meniscus surgery using the Watanabe arthroscope. Orthop Clin North Am 10:629-642

18. Jacquet C, Pujol N, Pauly V, Beaufils P, Ollivier M (2019) Analysis of the trends in arthroscopic meniscectomy and meniscus repair procedures in France from 2005 to 2017. Orthop Traumatol Surg Res 105:677-682

19. Kim ES, Omura PMC, Lo AW (2017) Accelerating biomedical innovation: a case study of the SPARK program at Stanford University, School of Medicine. Drug Discov Today 22:1064-1068

20. Kopf S, Beaufils P, Hirschmann MT, Rotigliano N, Ollivier M, Pereira H et al (2020) Management of traumatic meniscus tears: the 2019 ESSKA meniscus consensus. Knee Surg Sports Traumatol Arthrosc 28:1177-1194

21. Kou L, Xiao S, Sun R, Bao S, Yao Q, Chen R (2019) Biomaterial-engineered intra-articular drug delivery systems for osteoarthritis therapy. Drug Deliv 26:870-885

22. LaPrade RF, Geeslin AG, Murray IR, MusahI V, Zlotnicki JP, Petrigliano F et al (2016) Biologic treatments for sports injuries II think tank-current concepts, future research, and barriers to advancement, Part 1: biologics overview, ligament injury, tendinopathy. Am J Sports Med 44:3270-3283

23. Lee WQ, Gan JZ, Lie DTT (2019) Save the meniscus - clinical outcomes of meniscectomy versus meniscal repair. J Orthop Surg (Hong Kong) 27:2309499019849813

24. Li J, Lu D, Lin W, Li Q, Hu J, Xu D et al (2020) Prediction and potential preventions for the development of posttraumatic osteoarthritis after the terrible triad injury: a multicenter risk factors study. Biomed Res Int 2020:6508781
25. Li W, Zhang L, Ge X, Xu B, Zhang W, Qu L et al (2018) Microfluidic fabrication of microparticles for biomedical applications. Chem Soc Rev 47:5646-5683

26. Lubowitz JH, Poehling GG (2011) Save the meniscus. Arthroscopy 27:301-302

27. Luis E, Pan HM, Bastola AK, Bajpai R, Sing SL, Song J, et al. (2020) 3D printed silicone meniscus implants: influence of the 3D printing process on properties of pilicone pmplants. Polymers (Basel) 12:9

28. Milano G, Sánchez M, Jo CH, Saccomanno MF, Thampatty BP, Wang JHC (2019) Platelet-rich plasma in orthopaedic sports medicine: state of the art. JISAKOS 4:188-195

29. Mohanraj B, Duan G, Peredo A, Kim M, Tu F, Lee D, et al. (2019) Mechanically-activated microcapsules for 'On-Demand' drug delivery in dynamically loaded musculoskeletal tissues. Adv Funct Mater 29(15)

30. Mohanraj B, Hou C, Meloni GR, Cosgrove BD, Dodge GR, Mauck RL (2014) A high throughput mechanical screening device for cartilage tissue engineering. J Biomech 47:2130-2136

31. Moran CJ, Busilacchi A, Lee CA, Athanasiou KA, Verdonk PC (2015) Biological augmentation and tissue engineering approaches in meniscus surgery. Arthroscopy 31:944-955

32. Murray IR, Geeslin AG, Goudie EB, Petrigliano FA, LaPrade RF (2017) Minimum information for studies evaluating biologics in orthopaedics (MIBO): platelet-rich plasma and mesenchymal stem cells. J Bone Joint Surg Am 99:809-819

33. Murray IR, LaPrade RF, MusahI V, Geeslin AG, Zlotnicki JP, Mann BJ et al (2016) Biologic treatments for sports injuries II think tank-current concepts, future Research, and barriers to advancement, Part 2: rotator cuff. Orthop J Sports Med 4:2325967116636586

34. Murray IR, Makaram NS, Sherman SL, Safran MR, LaPrade RF, Abrams GD (2020) We need robust nomenclature for orthobiologics: letter to editor. Am J Sports Med 48:NP52-NP54

35. Nakama GY, Gonzalez S, Matre P, Mu X, Whitney KE, Utsunomiya H et al (2020) Effect of oral losartan on orthobiologics: implications for plateletrich plasma and bone marrow concentrate-A rabbit study. Int J Mol Sci 21(19):7374

36. Pujol N, Beaufils P (2019) Save the meniscus again! Knee Surg Sports Traumatol Arthrosc 27:341-342

37. Pujol N, Salle De Chou E, Boisrenoult P, Beaufils P (2015) Platelet-rich plasma for open meniscal repair in young patients: any benefit? Knee Surg Sports Traumatol Arthrosc 23:51-58

38. Rai MF, Pham CT (2018) Intra-articular drug delivery systems for joint diseases. Curr Opin Pharmacol 40:67-73

39. Rauschmann MA, Deb R, Thomann KD, Zichner L (2000) History of meniscus surgery. From excision of joint loose bodies to meniscus suture. Orthopade 29:1044-1054

40. Razi M, Mortazavi SMJ (2020) Save the Meniscus, A good Strategy to Preserve the Knee. Arch Bone Jt Surg 8:1-4

41. Riegger J, Brenner RE (2020) Pathomechanisms of posttraumatic osteoarthritis: chondrocyte behavior and fate in a precarious environment. Int J Mol Sci 21(5)

42. Rodeo SA, Bedi A (2020) 2019-2020 NFL and NFL physician society orthobiologics consensus statement. Sports Health 12:58-60

43. Samuelsen BT, Johnson NR, Hevesi M, Levy BA, Dahm DL, Stuart MJ et al (2018) Comparative outcomes of all-inside versus inside-out repair of bucket-handle meniscal tears: a propensity-matched analysis. Orthop J Sports Med 6:2325967118779045

44. Schroeder A, Rubin JP, Kokai L, Sowa G, Chen J, Onishi K (2020) Use of adipose-derived orthobiologics for musculoskeletal injuries: a narrative review. PM R 12:805-816

45. Seil $R$, Becker $R$ (2016) Time for a paradigm change in meniscal repair: save the meniscus! Knee Surg Sports Traumatol Arthrosc 24:1421-1423

46. Seil R, Karlsson J, Beaufils P, Becker R, Kopf S, Ollivier M et al (2017) The difficult balance between scientific evidence and clinical practice: 
the 2016 ESSKA meniscus consensus on the surgical management of degenerative meniscus lesions. Knee Surg Sports Traumatol Arthrosc 25:333-334

47. Sherman BJ, Chahla J, Glowney J, Frank RM (2019) The role of orthobiologics in the management of osteoarthritis and focal cartilage defects. Orthopedics 42:66-73

48. Sutton JB (1884) The nature of certain ligaments. J Anat Physiol 18:i2-238

49. Tuman J, Haro MS, Foley S, Diduch D (2012) All-inside meniscal repair devices and techniques. Expert Rev Med Devices 9:147-157

50. Watanabe M (1977) Development of arthroscope. Ryumachi 17:371-372

51. Zellner J, Hierl K, Mueller M, Pfeifer C, Berner A, Dienstknecht T et al (2013) Stem cell-based tissue-engineering for treatment of meniscal tears in the avascular zone. J Biomed Mater Res B Appl Biomater 101:1133-1142

52. Ziegler CG, Van Sloun R, Gonzalez S, Whitney KE, DePhillipo NN, Kennedy Ml et al (2019) Characterization of growth factors, cytokines, and chemokines in bone marrow concentrate and platelet-rich plasma: a prospective analysis. Am J Sports Med 47:2174-2187

\section{Publisher's Note}

Springer Nature remains neutral with regard to jurisdictional claims in published maps and institutional affiliations.

\section{Submit your manuscript to a SpringerOpen ${ }^{\odot}$ journal and benefit from:}

- Convenient online submission

- Rigorous peer review

- Open access: articles freely available online

- High visibility within the field

- Retaining the copyright to your article

Submit your next manuscript at $\boldsymbol{\nabla}$ springeropen.com 